\title{
On Laurent series with multiply positive coefficients
}

\author{
NATALYA A. ZHELTUKHINA
}

\begin{abstract}
A bstract. We consider the class of doubly infinite sequences $\left\{a_{k}\right\}_{k=-\infty}^{\infty}$ whose truncated sequences $\left\{a_{k}\right\}_{k=-n}^{n}$ are 3-times positive in the sense of Pólya and Fekete for all $n=1,2, \ldots$, and $a_{0} \neq 0$. We obtain a characterization of this class in terms of independent parameters. We also find an estimate of the growth order of the corresponding Laurent series $\sum_{k=-\infty}^{\infty} a_{k} z^{k}$.
\end{abstract}

\section{Introduction and results}

Let

$$
\left\{a_{n}\right\}_{n=-\infty}^{\infty}, \quad a_{0} \neq 0
$$

be a doubly infinite sequence, and

$$
f(z)=\sum_{n=-\infty}^{\infty} a_{n} z^{n}
$$

the corresponding generating Laurent series. Recall [5] that the sequence (1.1) is called totally positive if all minors of the four-way infinite matrix

$$
\left(\begin{array}{lllllllll}
\ldots & a_{-2} & a_{-1} & a_{0} & a_{1} & a_{2} & a_{3} & a_{4} & \ldots \\
\ldots & a_{-3} & a_{-2} & a_{-1} & a_{0} & a_{1} & a_{2} & a_{3} & \ldots \\
\ldots & a_{-4} & a_{-3} & a_{-2} & a_{-1} & a_{0} & a_{1} & a_{2} & \ldots \\
& & & & \ldots & & & &
\end{array}\right)
$$

are nonnegative. Denote by $P F_{\infty}$ the class of all totally positive sequences. In 1953, EDREI [1] found an exhaustive characterization of totally positive sequences (1.1) in terms of generating functions (1.2).

Edrei's Theorem (see [1], [2, p. 427]). A function $f(z)$ is a generating function of a totally positive sequence if and only if

$$
f(z)=C z^{k} \exp \left(q_{-1} z^{-1}+q_{1} z\right) \prod_{i=1}^{\infty} \frac{\left(1+\alpha_{i} z\right)\left(1+\delta_{i} z^{-1}\right)}{\left(1-\beta_{i} z\right)\left(1-\gamma_{i} z^{-1}\right)},
$$

Received June 12, 2001.

$0133-3852 / 04 / \$ 20.00$

(C) 2004 Akadémiai Kiadó, Budapest 
where $k$ is an integer and

$$
C>0, \quad q_{-1}, q_{1}, \alpha_{i}, \beta_{i}, \gamma_{i}, \delta_{i} \geq 0, \quad \sum_{i=1}^{\infty}\left(\alpha_{i}+\beta_{i}+\gamma_{i}+\delta_{i}\right)<\infty .
$$

In [6] Schoenberg generalized the concept of a totally positive sequence as follows: Let $r$ be a given natural number. We say that the sequence (1.1) is $r$-times positive, provided the matrix (1.3) has no negative minor of order $\leq r$.

Denote by $P F_{r}$ the class of all $r$-times positive sequences, $r \in \mathbf{N}$. If a Laurent series (1.2) is a generating function of an $r$-times positive sequence, we shall write $f \in P F_{r}$. Evidently, $P F_{1} \supset P F_{2} \supset P F_{3} \supset \cdots \supset$ $P F_{\infty}$. Clearly, the class $P F_{1}$ consists of all sequences (1.1) with nonnegative coefficients. It is a simple matter to see that the class $P F_{2}$ consists of all sequences of the form

$$
a_{n}=\exp \{-\psi(n)\}, \quad n \in \mathbf{Z},
$$

where $\psi: \mathbf{Z} \rightarrow(-\infty ;+\infty], \psi(0)<\infty$, is a convex function. The class $P F_{\infty}$ is characterized by Edrei's Theorem. The problem of the description of the classes $P F_{r}, 3 \leq r<\infty$, is at present far from being solved.

In view of Edrei's Theorem, SchoENBERG [6] stated the problem of discovering analytical properties of the generating function (1.2) of an $r$ times positive sequence (1.1). He considered finite $r$-times positive sequences

$$
\left(\ldots, 0,0, a_{0}, a_{1}, \ldots, a_{m}, 0,0, \ldots\right),
$$

and described the zero distribution of the corresponding generating polynomials. Here we restrict ourselves to some subclasses of $P F_{r}, 3 \leq r \leq \infty$, containing infinite sequences. Mostly, we deal with the case $r=3$.

Denote by $T Q_{r}, r \in \mathbf{N} \cup\{\infty\}$, the class of all sequences (1.1) such that all truncated sequences

$$
\left\{a_{k}\right\}_{k=-n}^{n}:=\left\{\ldots, 0,0, a_{-n}, a_{-n+1}, \ldots, a_{n}, 0,0, \ldots\right\}, \quad n=1,2, \ldots,
$$

are $r$-times positive. Their subclasses $Q_{r} \subset T Q_{r}$ consisting of all one-side sequences (with $a_{n}=0$ for $n<0$ ) were considered in [3] and [4]. We shall reduce the problem of characterization of the class $T Q_{3}$ to that of $Q_{3}$. First, we present some known facts concerning $Q_{3}$.

Theorem A (see [3]). If a formal power series

$$
f(z)=\sum_{k=0}^{\infty} a_{k} z^{k}
$$


belongs to $Q_{n}$ for some $n \geq 3$, then it converges on the whole complex plane $\mathbf{C}$ and its sum $f(z)$ is an entire function of order 0. Moreover,

$$
\limsup _{r \rightarrow \infty} \frac{\log M(r, f)}{(\log r)^{2}} \leq \frac{1}{2 \log \frac{1+\sqrt{5}}{2}}, \quad \text { where } M(r, f)=\max \{f(z):|z|=r\} \text {. }
$$

There is a characterization of class $Q_{3}$ in terms of independent parameters. The role of independent parameters is played by the points of the set $(0, \infty) \times[0, \infty) \times U$, where

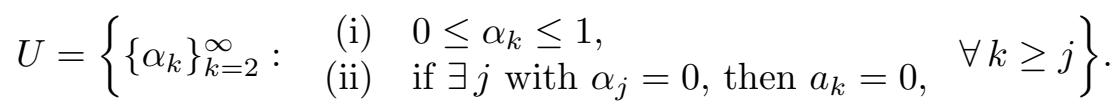

Define the numbers

$$
\begin{gathered}
\left(1.6^{\prime}\right)\left[\alpha_{2}\right]=1+\alpha_{2}, \quad\left[\alpha_{2} \alpha_{3}\right]=1+\alpha_{3} \sqrt{\left[\alpha_{2}\right]}, \quad\left[\alpha_{2} \alpha_{3} \alpha_{4}\right]=1+\alpha_{4} \sqrt{\left[\alpha_{2} \alpha_{3}\right]}, \\
\ldots, \quad\left[\alpha_{2} \alpha_{3} \ldots \alpha_{n}\right]=1+\alpha_{n} \sqrt{\left[\alpha_{2} \alpha_{3} \ldots \alpha_{n-1}\right]}, \quad \ldots
\end{gathered}
$$

if

Theorem B (see [4]). A power series (1.5) belongs to $Q_{3}$ if and only

$$
a_{1}=a_{0} \alpha, \quad a_{n}=\frac{a_{0} \alpha^{n} \alpha_{2}^{n-1} \alpha_{3}^{n-2} \cdots \alpha_{n-1}^{2} \alpha_{n}}{\left[\alpha_{2}\right]^{n / 2}\left[\alpha_{2} \alpha_{3}\right]^{(n-1) / 2} \cdots\left[\alpha_{2} \alpha_{3} \cdots \alpha_{n-1}\right]^{3 / 2}\left[\alpha_{2} \alpha_{3} \cdots \alpha_{n}\right]},
$$

where

$$
a_{0}>0, \quad \alpha \geq 0, \quad\left\{\alpha_{k}\right\}_{k=2}^{\infty} \in U
$$

and $U$ is defined in (1.6).

Since $T Q_{3}$ is a subclass of $P F_{2}$, any sequence (1.1) in $T Q_{3}$ admits representation (1.4). Set

$$
\begin{array}{cl}
N_{1}=\min \{n \geq 1: \psi(n)=+\infty\} & \left(N_{1}=+\infty \text { if } \psi(n)<+\infty, \forall n \geq 1\right), \\
N_{2}=\max \{n \leq-1: \psi(n)=+\infty\} & \left(N_{2}=-\infty \text { if } \psi(n)<+\infty, \forall n \leq-1\right),
\end{array}
$$
and

$$
\Delta_{2} \psi(n):= \begin{cases}\psi(n)-2 \psi(n-1)+\psi(n-2) & \text { if } 1 \leq n<N_{1} \\ +\infty & \text { if } n \geq N_{1} \text { or } n \leq N_{2}, \\ \psi(n)-2 \psi(n+1)+\psi(n+2) & \text { if } N_{2}<n \leq-1\end{cases}
$$

Define the sequence $\left\{\omega_{n}\right\}_{n=2}^{\infty}$ as follows:

$$
\omega_{2}=1, \quad \omega_{n}=\left[\alpha_{2} \alpha_{3} \cdots \alpha_{n-1}\right]_{\alpha_{2}=\alpha_{3}=\cdots=\alpha_{n-1}=1}, \quad n \geq 3 .
$$

Theor em C (see [4]). For a formal power series (1.5) to belong to $Q_{3}$ it is necessary and sufficient that

$$
\Delta_{2} \psi(n) \geq \log \left(1+\frac{1}{\sqrt{\omega_{n}}}\right), \quad n \geq 2,
$$

where $\omega_{n}, n \geq 2$, are defined in (1.7). 
Our main result allowing to reduce double-sided sequences to one-sided ones is stated in the following theorem.

Theorem 1. A Laurent series (1.2) belongs to the class $T Q_{3}$ if and only if both power series

$$
f_{1}(z)=\sum_{k=0}^{\infty} a_{k-1} z^{k} \quad \text { and } \quad f_{2}(z)=\sum_{k=0}^{\infty} a_{1-k} z^{k}
$$

belong to the class $Q_{3}$.

It is natural to ask whether the class $P F_{3}$ itself satisfies the property: both power series in Theorem 1 belong to the class $P F_{3}$. The answer is negative as the following example shows. The Laurent series

$$
f(z)=\sum_{n=-\infty}^{\infty} q^{-n^{2}} z^{n}
$$

belongs to $P F_{\infty}$ for any $q>1$ (see [2, p. 433]). However, by the identity

$$
\begin{gathered}
I_{k}:=\left|\begin{array}{lll}
q^{-(k+1)^{2}} & q^{-(k+2)^{2}} & q^{-(k+3)^{2}} \\
q^{-k^{2}} & q^{-(k+1)^{2}} & q^{-(k+2)^{2}} \\
0 & q^{-k^{2}} & q^{-(k+1)^{2}}
\end{array}\right|= \\
=q^{-(k+1)^{2}-(k+2)^{2}-(k+3)^{2}+6 k+5}\left(q^{6}-2 q^{4}+1\right), \quad k \in \mathbf{Z},
\end{gathered}
$$

we conclude that $I_{k}<0$ for $1<q^{2}<\frac{1+\sqrt{5}}{2}$, and hence it follows that

$$
\sum_{n=k}^{\infty} q^{-n^{2}} z^{n} \notin P F_{3} \quad \text { for } 1<q^{2}<\frac{1+\sqrt{5}}{2} \text { for any } k \in \mathbf{Z} .
$$

Theorems 1 and A allow us to derive the following estimates on the growth order of functions in $T Q_{3}$.

Theor em 2. Let a Laurent series (1.2) belong to $T Q_{r}$ for some $r \geq 3$. Then it converges in $\mathbf{C} \backslash\{0\}$ and

$$
\begin{aligned}
& \limsup _{r \rightarrow \infty} \frac{\log M(r, f)}{(\log r)^{2}} \leq \frac{1}{2 \log \frac{1+\sqrt{5}}{2}}, \\
& \limsup _{r \rightarrow 0} \frac{\log M(r, f)}{\left(\log \frac{1}{r}\right)^{2}} \leq \frac{1}{2 \log \frac{1+\sqrt{5}}{2}} .
\end{aligned}
$$

where $M(r, f)=\max \{f(z):|z|=r\}$.

Combining Theorems 1 and $\mathrm{B}$, we deduce a representation of the class $T Q_{3}$ in terms of independent parameters. 
Theorem 3. A Laurent series (1.2) belongs to $T Q_{3}$ if and only if

$$
a_{0}=a_{-1} \alpha,
$$

$$
a_{n-1}=\frac{a_{-1} \alpha^{n} \alpha_{2}^{n-1} \alpha_{3}^{n-2} \cdots \alpha_{n-1}^{2} \alpha_{n}}{\left[\alpha_{2}\right]^{n / 2}\left[\alpha_{2} \alpha_{3}\right]^{(n-1) / 2} \cdots\left[\alpha_{2} \alpha_{3} \cdots \alpha_{n-1}\right]^{3 / 2}\left[\alpha_{2} \alpha_{3} \cdots \alpha_{n}\right]}, \quad n \geq 2,
$$

$$
a_{-n+1}=\frac{a_{-1} \alpha \beta^{n-1} \beta_{2}^{n-1} \beta_{3}^{n-2} \cdots \beta_{n-1}^{2} \beta_{n}}{\left[\beta_{2}\right]^{n / 2}\left[\beta_{2} \beta_{3}\right]^{(n-1) / 2} \cdots\left[\beta_{2} \beta_{3} \cdots \beta_{n-1}\right]^{3 / 2}\left[\beta_{2} \beta_{3} \cdots \beta_{n}\right]}, \quad n \geq 2,
$$

where $U$ is defined in (1.6) and

$$
\alpha_{-1}>0, \alpha>0,\left\{\alpha_{k}\right\}_{k=2}^{\infty} \in U, \beta=\frac{1+\alpha_{2}}{\alpha \alpha_{2}}, \beta_{2}=\alpha_{2},\left\{\beta_{k+1}\right\}_{k=2}^{\infty} \in U .
$$

Since $T Q_{3} \subset P F_{3}$, Theorem 3 provides a rich source of functions from $P F_{3}$. The important point to note here is that Theorem 3 allows us to construct functions in $P F_{3} \backslash P F_{4}$. The problem of finding the greatest $n \geq 3$, such that $f(z) \in P F_{n}$ for some special functions $f(z)$, was treated in $([2$, Chapter $8, \S 12])$.

Corollary. Let $U$ be defined in (1.6). For any $\alpha_{2}, \frac{\sqrt{5}-1}{2}<\alpha_{2} \leq 1$, there exist $\alpha_{3}, \beta_{3}, 0<\alpha_{3}, \beta_{3} \leq 1$, such that for all $\left\{\beta_{k+2}\right\}_{k=2}^{\infty} \in U$ and $\left\{\alpha_{k+2}\right\}_{k=2}^{\infty} \in U$, the sequence (1.1) defined in (1.10) and (1.11) belongs to $\mathrm{PF}_{3} \backslash \mathrm{PF}_{4}$.

The next theorem is an immediate consequence of Theorems 1 and C.

Theorem 4. For a sequence (1.4) to belong to $T Q_{3}$ it is nessesary and sufficient that

$$
\Delta_{2} \psi(n) \geq \log \left(1+\frac{1}{\sqrt{\omega_{n+1}}}\right), \quad n \geq 1
$$

and

$$
\Delta_{2} \psi(n) \geq \log \left(1+\frac{1}{\sqrt{\omega_{-n+1}}}\right), \quad n \leq-1 .
$$

For a sequence (1.4) to be a $P F_{2}$-sequence, we must have the convexity of the function $\psi: \mathbf{Z} \rightarrow(-\infty ; \infty], \psi(0)<\infty$, that is, nonnegativity of $\Delta_{2} \psi(n)$. Theorem 4 demonstrates how the nonnegativity changes if we require (1.4) to belong to $T Q_{3} \subset P F_{2}$. 


\section{Proof of Theorem 1}

Lemma 1.1. Let a Laurent series (1.2) belong to $T Q_{2}$. Set

$$
k_{1}=\min \left\{k>0: a_{k}=0\right\}, \quad \text { and } \quad k_{2}=\max \left\{k<0: a_{k}=0\right\} .
$$

Then $a_{k}=0$ for all $k<k_{2}+1$ and $k>k_{1}-1$.

Proof. Let us show that $a_{k}=0$ for all $k \leq k_{2}$. Assume $k_{2}>-\infty$. We have

$$
\left|\begin{array}{ll}
a_{k+1} & a_{k_{2}+1} \\
a_{k} & a_{k_{2}}
\end{array}\right|=-a_{k} a_{k_{2}+1} \geq 0
$$

for all $k<k_{2}$. Hence, $a_{k}=0$ for all $k \leq k_{2}$. That $a_{k}=0$ for all $k \geq k_{1}$ can be proved similarly.

Without loss of generality we may assume that $a_{2} \neq 0$ and $a_{-2} \neq 0$, that is, $k_{1}>2$ and $k_{2}<-2$. Lemma 1.1 allows us to introduce the positive numbers

$$
\delta_{k}= \begin{cases}a_{k-1}^{2} /\left(a_{k} a_{k-2}\right) & \text { if } 0<k<k_{1} \\ a_{k+1}^{2} /\left(a_{k} a_{k+2}\right) & \text { if } k_{2}<k<0\end{cases}
$$

Note that $\delta_{1}=\delta_{-1}$.

Lemma 1.2. Let the Laurent series (1.2) belong to TQ $Q_{3}$. Then both

$$
f_{1}(z)=\sum_{k=0}^{\infty} a_{k-1} z^{k} \quad \text { and } \quad f_{2}(z)=\sum_{k=0}^{\infty} a_{1-k} z^{k}
$$

belong to $Q_{3}$.

Proof. (i) Let us show that $f_{1}(z)$ belongs to $Q_{3}$. We shall use the following test of $m$-times positivity, which is due to SCHONBERG [6].

Theorem (see [6]). Let $\left\{b_{k}\right\}_{k=0}^{n}$ be a finite sequence of numbers. Consider the matrices

$$
B_{k}=\left(\begin{array}{lllllllll}
b_{0} & b_{1} & b_{2} & \ldots & b_{n} & 0 & 0 & \ldots & 0 \\
0 & b_{0} & b_{1} & \ldots & b_{n-1} & b_{n} & 0 & \ldots & 0 \\
0 & 0 & b_{0} & \ldots & b_{n-2} & b_{n-1} & b_{n} & \ldots & 0 \\
. & . & . & \ldots & . & . & . & \ldots & . \\
0 & 0 & 0 & \ldots & . & . & . & \ldots & b_{n}
\end{array}\right) \quad k=1,2, \ldots, m
$$

where $B_{k}$ consists of $k$ rows and $n+k$ columns. Assume that the following condition is satisfied for $k=1,2, \ldots, m$ : all $k \times k$-minors of $B_{k}$ consisting of consecutive columns are strictly positive. Then $\left\{\ldots, 0, b_{0}, b_{1}, \ldots, b_{n}, 0, \ldots\right\}$ is an m-times positive sequence. 
Fix any $n, 1<n<k_{1}$, and consider the following three matrices:

$$
\begin{gathered}
A_{1}=\left(\begin{array}{lllllll}
a_{-1} & a_{0} & a_{1} & \ldots & a_{n}-\varepsilon
\end{array}\right), \\
A_{2}=\left(\begin{array}{llllllll}
a_{-1} & a_{0} & a_{1} & \ldots & a_{n}-\varepsilon & 0 \\
0 & a_{-1} & a_{0} & \ldots & a_{n-1} & a_{n}-\varepsilon
\end{array}\right), \\
A_{3}=\left(\begin{array}{llllllll}
a_{-1} & a_{0} & a_{1} & \ldots & a_{n}-\varepsilon & 0 & 0 \\
0 & a_{-1} & a_{0} & \ldots & a_{n-1} & a_{n}-\varepsilon & 0 \\
0 & 0 & a_{-1} & \ldots & a_{n-2} & a_{n-1} & a_{n}-\varepsilon
\end{array}\right) .
\end{gathered}
$$

All minors of $A_{1}$ are positive for $0<\varepsilon<a_{n}$. For all $m \in \mathbf{N} \cap\{m \leq n\}$,

$$
\left|\begin{array}{lll}
a_{-m+1} & a_{m} & 0 \\
a_{-m} & a_{m-1} & a_{m} \\
0 & a_{m-2} & a_{m-1}
\end{array}\right|=a_{-m+1} a_{m-2} a_{m}\left(\delta_{m}-1-\frac{a_{m-1} a_{-m}}{a_{m-2} a_{-m+1}}\right) \geq 0
$$

whence $\delta_{m}>1, m \in \mathbf{N} \cap\{m \leq n\}$. Therefore, all $2 \times 2$ minors of $A_{2}$ consisting of consecutive columns

$$
\begin{gathered}
\left|\begin{array}{ll}
a_{k} & a_{k+1} \\
a_{k-1} & a_{k}
\end{array}\right|=a_{k-1} a_{k+1}\left(\delta_{k+1}-1\right), \quad 0 \leq k \leq n-1, \\
\left|\begin{array}{ll}
a_{-1} & a_{0} \\
0 & a_{-1}
\end{array}\right|, \quad\left|\begin{array}{ll}
a_{n}-\varepsilon & 0 \\
a_{n-1} & a_{n}-\varepsilon
\end{array}\right|
\end{gathered}
$$

are strictly positive.

Consider all $3 \times 3$ minors of $A_{3}$ consisting of consecutive columns:

$$
\begin{gathered}
M_{-1}=\left|\begin{array}{lll}
a_{-1} & a_{0} & a_{1} \\
0 & a_{-1} & a_{0} \\
0 & 0 & a_{-1}
\end{array}\right|>0, \quad M_{0}=\left|\begin{array}{lll}
a_{0} & a_{1} & a_{2} \\
a_{-1} & a_{0} & a_{1} \\
0 & a_{-1} & a_{0}
\end{array}\right|, \\
M_{k}=\left|\begin{array}{lll}
a_{k} & a_{k+1} & a_{k+2}(-\varepsilon) \\
a_{k-1} & a_{k} & a_{k+1} \\
a_{k-2} & a_{k-1} & a_{k}
\end{array}\right|, \quad 1 \leq k \leq n-2, \\
M_{n-1}(\varepsilon)=\left|\begin{array}{lll}
a_{n-1} & a_{n}-\varepsilon & 0 \\
a_{n-2} & a_{n-1} & a_{n}-\varepsilon \\
a_{n-3} & a_{n-2} & a_{n-1}
\end{array}\right|, \\
M_{n}(\varepsilon)=\left|\begin{array}{lll}
a_{n}-\varepsilon & 0 & 0 \\
a_{n-1} & a_{n}-\varepsilon & 0 \\
a_{n-2} & a_{n-1} & a_{n}-\varepsilon
\end{array}\right|>0 .
\end{gathered}
$$

Since $\left\{\ldots, 0,0, a_{-k-1}, a_{-k}, \ldots, a_{k}, a_{k+1}, \ldots\right\}$ are 3 -times positive, and $\delta_{k}>1$ for all $1 \leq k \leq n-2$, we have

$$
M_{0}=\left|\begin{array}{lll}
a_{0} & a_{1} & 0 \\
a_{-1} & a_{0} & a_{1} \\
0 & a_{-1} & a_{0}
\end{array}\right|+a_{2} a_{-1}^{2}>0
$$


and

$$
\begin{gathered}
M_{k} \geq\left|\begin{array}{lll}
a_{k} & a_{k+1} & a_{k+2}(-\varepsilon) \\
a_{k-1} & a_{k} & a_{k+1} \\
a_{k-2} & a_{k-1} & a_{k}
\end{array}\right|= \\
=\left|\begin{array}{lll}
a_{k} & a_{k+1} & 0 \\
a_{k-1} & a_{k} & a_{k+1} \\
a_{k-2} & a_{k-1} & a_{k}
\end{array}\right|+\left(a_{k+2}(-\varepsilon)\right) a_{k-2} a_{k}\left(\delta_{k}-1\right)>0
\end{gathered}
$$

for all sufficiently small $\varepsilon$.

We have

$$
\begin{aligned}
& M_{n-1}(\varepsilon)=a_{n-1}^{3}+\left(a_{n}-\varepsilon\right)^{2} a_{n-3}-2\left(a_{n}-\varepsilon\right) a_{n-1} a_{n-2}, \\
& M_{n-1}(0) \geq 0, \quad M^{\prime}{ }_{n-1}(0)=2 a_{n} a_{n-3}\left(\delta_{n-1} \delta_{n}-1\right)>0 .
\end{aligned}
$$

Hence, $M_{n-1}(\varepsilon)>0$ for all sufficiently small $\varepsilon>0$. So, all $3 \times 3$ minors of $A_{3}$ consisting of consecutive columns are strictly positive. By Schoenberg's theorem stated above, it means that $\left\{\ldots, 0,0, a_{-1}, \ldots, a_{n-1}, a_{n}-\varepsilon, 0,0, \ldots\right\}$ is a 3 -times positive sequence for all sufficiently small $\varepsilon$. Taking the limit as $\varepsilon$ tends to 0 , we have that $\left\{\ldots 0,0, a_{-1}, \ldots, a_{n-1}, a_{n}, 0,0, \ldots\right\}$ is a 3 -times positive sequence for all $n \in \mathbf{N}$. To prove that $f_{1}(z) \in Q_{3}$, it suffices to prove that $\left\{\ldots, 0,0, a_{-1}, a_{0}, 0,0, \ldots\right\}$ and $\left\{\ldots, 0,0, a_{-1}, a_{0}, a_{1}, 0,0, \ldots\right\}$ are 3 -times positive sequences. But the first sequence is even totally positive. And the second one is 3-times positive, that follows from $f \in T Q_{3}$.

(ii) Note that $f \subset T Q_{3}$ implies $f(1 / z) \in T Q_{3}$. Then, by part (i), $f_{2}(z) \in Q_{3}$.

Lemma 1.3. Let the Laurent series

$$
f_{1}(z)=\sum_{k=0}^{\infty} a_{k-1} z^{k} \quad \text { and } \quad f_{2}(z)=\sum_{k=0}^{\infty} a_{1-k} z^{k}
$$

both belong to $Q_{3}$. Then the Laurent series (1.2) belongs to $T Q_{3}$.

Proof. Fix any $n>1$. Let $k_{1}$ and $k_{2}$ be as in Lemma 1.1. Denote

$$
\begin{gathered}
n_{1}=\left\{\begin{array}{lll}
n & \text { if } k_{1}=\infty, \\
\min \left\{n, k_{1}-1\right\} & \text { if } k_{1}<\infty ;
\end{array}\right. \\
n_{2}=\left\{\begin{array}{lll}
-n & \text { if } k_{2}=-\infty, \\
\max \left\{-n, k_{2}+1\right\} & \text { if } k_{2}>-\infty .
\end{array}\right.
\end{gathered}
$$

Let us prove that

$$
\left\{\ldots, 0,0, a_{n_{2}}-\varepsilon, a_{n_{2}+1}, \ldots, a_{n_{1}-1}, a_{n_{1}}-\varepsilon, 0,0, \ldots\right\}
$$

is a 3 -times positive sequence for any sufficiently small $\varepsilon$. Consider the following three matrices:

$$
A_{1}=\left(\begin{array}{lllll}
a_{n_{2}}-\varepsilon & a_{n_{2}+1} & \ldots & a_{n_{1}-1} & a_{n_{1}}-\varepsilon
\end{array}\right),
$$




$$
\begin{gathered}
A_{2}=\left(\begin{array}{lllll}
a_{n_{2}}-\varepsilon & a_{n_{2}+1} & \ldots & a_{n_{1}}-\varepsilon & 0 \\
0 & a_{n_{2}}-\varepsilon & \ldots & a_{n_{1}-1} & a_{n_{1}}-\varepsilon
\end{array}\right) \\
A_{3}=\left(\begin{array}{lllllll}
a_{n_{2}}-\varepsilon & a_{n_{2}+1} & a_{n_{2}+2} & \ldots & a_{n_{1}}-\varepsilon & 0 & 0 \\
0 & a_{n_{2}}-\varepsilon & a_{n_{2}+1} & \ldots & a_{n_{1}-1} & a_{n_{1}}-\varepsilon & 0 \\
0 & 0 & a_{n_{2}}-\varepsilon & \ldots & a_{n_{1}-2} & a_{n_{1}-1} & a_{n_{1}}-\varepsilon
\end{array}\right) .
\end{gathered}
$$

All minors of $A_{1}$ are strictly positive for $0<\varepsilon<\min \left\{a_{n_{1}}, a_{n_{2}}\right\}$. For $1 \leq m \leq n_{1}$,

$$
\left|\begin{array}{lll}
a_{0} & a_{m} & 0 \\
a_{-1} & a_{m-1} & a_{m} \\
0 & a_{m-2} & a_{m-1}
\end{array}\right|=a_{0} a_{m-2} a_{m}\left(\delta_{m}-1-\frac{a_{-1} a_{m-1}}{a_{0} a_{m-2}}\right) \geq 0 .
$$

So, $\delta_{m}>1$ for all $m, 1 \leq m \leq n_{1}$. Similarly, $\delta_{m}>1$ for all $m$, $n_{2} \leq m \leq-1$. Therefore, all $2 \times 2$ minors of $A_{2}$ consisting of consecutive columns:

$$
\begin{gathered}
\left|\begin{array}{ll}
a_{n_{2}}-\varepsilon & a_{n_{2}+1} \\
0 & a_{n_{2}}-\varepsilon
\end{array}\right|, \quad\left|\begin{array}{ll}
a_{k-1} & a_{k} \\
a_{k-2} & a_{k-1}
\end{array}\right|=a_{k} a_{k-2}\left(\delta_{k}-1\right), \quad 1 \leq k \leq n_{1}, \\
\left|\begin{array}{ll}
a_{k-1} & a_{k} \\
a_{k-2} & a_{k-1}
\end{array}\right|=a_{k} a_{k-2}\left(\delta_{k-2}-1\right), \quad n_{2}+2 \leq k \leq 1, \quad\left|\begin{array}{ll}
a_{n_{1}}-\varepsilon & 0 \\
a_{n_{1}-1} & a_{n_{1}}-\varepsilon
\end{array}\right|
\end{gathered}
$$

are strictly positive. Consider all $3 \times 3$ minors of $A_{3}$ consisting of consecutive columns:

$$
\begin{gathered}
M_{n_{2}+1}(\varepsilon)=\left|\begin{array}{lll}
a_{n_{2}+1} & a_{n_{2}+2} & a_{n_{2}+3} \\
a_{n_{2}}-\varepsilon & a_{n_{2}+1} & a_{n_{2}+2} \\
0 & a_{n_{2}}-\varepsilon & a_{n_{2}+1}
\end{array}\right|, \\
M_{n_{1}-1}(\varepsilon)=\left|\begin{array}{lll}
a_{n_{1}-1} & a_{n_{1}}-\varepsilon & 0 \\
a_{n_{1}-2} & a_{n_{1}-1} & a_{n_{1}}-\varepsilon \\
a_{n_{1}-3} & a_{n_{1}-2} & a_{n_{1}-1}
\end{array}\right|, \\
M_{k}=\left|\begin{array}{lll}
a_{k} & a_{k+1} & a_{k+2} \\
a_{k-1} & a_{k} & a_{k+1} \\
a_{k-2} & a_{k-1} & a_{k}
\end{array}\right|, \quad n_{2}+2 \leq k \leq n_{1}-2 .
\end{gathered}
$$

Since $f_{1}(z) \in Q_{3}$, for $k \geq 1$ we have

$$
M_{k}>\left|\begin{array}{lll}
a_{k} & a_{k+1} & 0 \\
a_{k-1} & a_{k} & a_{k+1} \\
a_{k-2} & a_{k-1} & a_{k}
\end{array}\right| \geq 0 .
$$

Since $f_{2}(z) \in Q_{3}$, for $k \leq-1$ we have

$$
M_{k}>\left|\begin{array}{lll}
a_{k} & a_{k+1} & a_{k+2} \\
a_{k-1} & a_{k} & a_{k+1} \\
0 & a_{k-1} & a_{k}
\end{array}\right| \geq 0
$$


Since $f_{1}(z) \in Q_{3}$, we also have

$$
M_{0}=\left|\begin{array}{lll}
a_{0} & a_{1} & a_{2} \\
a_{-1} & a_{0} & a_{1} \\
a_{-2} & a_{-1} & a_{0}
\end{array}\right|>\left|\begin{array}{lll}
a_{0} & a_{1} & a_{2} \\
a_{-1} & a_{0} & a_{1} \\
0 & a_{-1} & a_{0}
\end{array}\right| \geq 0 .
$$

The claim that $M_{n_{1}-1}(\varepsilon)$ and $M_{n_{2}+1}(\varepsilon)$ are strictly positive for all sufficiently small $\varepsilon>0$ one can prove by the same method as in Lemma 1.2. So, all $3 \times 3$ minors of $A_{3}$ consisting of consecutive columns are strictly positive. It follows from Schoenberg's theorem, stated at the beginning of the Proof of Lemma 1.2 that

$$
\left\{\ldots, 0,0, a_{n_{2}}-\varepsilon, a_{n_{2}+1}, \ldots, a_{n_{1}-1}, a_{n_{1}}-\varepsilon, 0,0, \ldots\right\}
$$

is a 3 -times positive sequence for any sufficiently small $\varepsilon>0$. Taking the limit as $\varepsilon$ tends to 0 , we find that

$$
\left\{\ldots 0,0, a_{n_{2}}, a_{n_{2}+1}, \ldots, a_{n_{1}-1}, a_{n_{1}}, 0,0, \ldots\right\}
$$

is a 3 -times positive sequence.

\section{Proof of Theorems 2, 3 and Corollary}

Proof of Theorem 2. Consider

$$
f_{1}(z)=\sum_{k=0}^{\infty} a_{k-1} z^{k} \quad \text { and } \quad f_{2}(z)=\sum_{k=0}^{\infty} a_{1-k} z^{k} .
$$

By Lemma 1.2, both $f_{1}(z)$ and $f_{2}(z)$ belong to $Q_{3}$. Applying Theorem A to $f_{1}(z)$ and $f_{2}(z)$, we have

$$
\limsup _{r \rightarrow \infty} \frac{\log M\left(r, f_{i}\right)}{(\log r)^{2}} \leq \frac{1}{2 \log c}, \quad c=\frac{1+\sqrt{5}}{2}, i=1,2 .
$$

Hence, the Laurent series

$$
f(z)=\sum_{k=-\infty}^{\infty} a_{k} z^{k}=\frac{1}{z} f_{1}(z)+z f_{2}\left(\frac{1}{z}\right)-\frac{a_{-1}}{z}-a_{0}-a_{1} z
$$

converges in $\mathbf{C} \backslash\{0\}$ and estimates (1.8) and (1.9) hold.

Proof of Theorem 3. (i) Let $f \in T Q_{3}$. Then by Lemma 1.2,

$$
\sum_{k=0}^{\infty} a_{k-1} z^{k} \in Q_{3}
$$

and Theorem B gives representation (1.10). Also, by Lemma 1.2,

$$
\sum_{k=0}^{\infty} a_{-k+1} z^{k} \in Q_{3}
$$


and Theorem B gives

$$
a_{-n+1}=\frac{a_{1} \beta^{n} \beta_{2}^{n-1} \beta_{3}^{n-2} \cdots \beta_{n-1}^{2} \beta_{n}}{\left[\beta_{2}\right]^{n / 2}\left[\beta_{2} \beta_{3}\right]^{(n-1) / 2} \cdots\left[\beta_{2} \beta_{3} \cdots \beta_{n-1}\right]^{3 / 2}\left[\beta_{2} \beta_{3} \cdots \beta_{n}\right]}, \quad n \geq 2 .
$$

To prove (1.11), it remains to prove that

$$
\beta=\frac{1+\alpha_{2}}{\alpha \alpha_{2}}, \quad \beta_{2}=\alpha_{2} .
$$

The formula (1.10) for the coefficient $a_{1}$ implies

$$
\frac{a_{-1}}{a_{1}}=\frac{1+\alpha_{2}}{\alpha_{2} \alpha^{2}} .
$$

Formulas (1.10) and (3.1) for the coefficient $a_{0}$ give

$$
a_{-1} \alpha=a_{1} \beta, \quad \text { that is, } \quad \beta=\frac{a_{-1}}{a_{1}} \alpha=\frac{1+\alpha_{2}}{\alpha_{2} \alpha} .
$$

It follows from $\delta_{1}=\delta_{-1}$ that $\alpha_{2}=\beta_{2}$.

(ii) Consider the Laurent series

$$
g_{1}(z)=\frac{a_{-1}}{z}+a_{-1} \alpha+\sum_{n=2}^{\infty} a_{n-1} z^{n-1},
$$

where $a_{-1}>0, \alpha \geq 0$ and the coefficients $a_{n-1}, n \geq 2$, are given in (1.10). By Theorem B, the sequence $\left\{a_{k}\right\}_{k=-1}^{\infty}$ belongs to $Q_{3}$.

Consider the Laurent series

$$
g_{2}(z)=a_{1} z^{-1}+a_{1} \beta+\sum_{n=2}^{\infty} a_{-n+1} z^{n-1},
$$

where

$$
a_{1}=\frac{a_{-1} \alpha^{2} \alpha_{2}}{1+\alpha_{2}}, \quad \beta=\frac{1+\alpha_{2}}{\alpha \alpha_{2}}, \quad \beta_{2}=\alpha_{2},
$$

and the coefficients $a_{-n+1}, n \geq 2$, are given in (1.11). We have $a_{1} \beta=a_{-1} \alpha$ and hence,

$$
\begin{gathered}
a_{0}=a_{-1} \alpha=a_{1} \beta \\
a_{-n+1}=\frac{a_{1} \beta^{n} \beta_{2}^{n-1} \beta_{3}^{n-2} \cdots \beta_{n-1}^{2} \beta_{n}}{\left[\beta_{2}\right]^{n / 2}\left[\beta_{2} \beta_{3}\right]^{(n-1) / 2} \cdots\left[\beta_{2} \beta_{3} \cdots \beta_{n-1}\right]^{3 / 2}\left[\beta_{2} \beta_{3} \cdots \beta_{n}\right]}, \quad n \geq 2 .
\end{gathered}
$$

By Theorem B, the sequence $\left\{a_{-k}\right\}_{k=-1}^{\infty}$ belongs to $Q_{3}$. By Theorem 1,

$$
f(z)=\sum_{k=-\infty}^{\infty} a_{k} z^{k} \in T Q_{3}
$$


Proof of Corollary. For a given $\alpha>0$ and $\frac{\sqrt{5}-1}{2}<\alpha_{2} \leq 1$, define the coefficients $a_{0}, a_{1}, a_{-1}, a_{2}, a_{-2}$ by formulas in (1.10) and (1.11). Taking appropriate $\alpha_{n}$ and $\beta_{n}, n \geq 3$, in formulas (1.10) and (1.11), we shall define $a_{n},|n| \geq 3$, such that

$$
f(z)=\sum_{k=-\infty}^{\infty} a_{k} z^{k} \in P F_{3} \backslash P F_{4} .
$$

Since, by (1.10) and (1.11),

$$
\begin{gathered}
\left|\begin{array}{llll}
a_{0} & a_{1} & a_{2} & a_{3} \\
a_{-1} & a_{0} & a_{1} & a_{2} \\
a_{-2} & a_{-1} & a_{0} & a_{1} \\
a_{-3} & a_{-2} & a_{-1} & a_{0}
\end{array}\right| \leq\left|\begin{array}{llll}
a_{0} & a_{1} & a_{2} & 0 \\
a_{-1} & a_{0} & a_{1} & a_{2} \\
a_{-2} & a_{-1} & a_{0} & a_{1} \\
0 & a_{-2} & a_{-1} & a_{0}
\end{array}\right|= \\
=a_{-1}^{4}\left|\begin{array}{llll}
\alpha & \frac{\alpha^{2} \alpha_{2}}{\left[\alpha_{2}\right]} & \frac{\alpha^{3} \alpha_{2}^{2} \alpha_{3}}{\left[\alpha_{2}\right]^{3 / 2}\left[\alpha_{2} \alpha_{3}\right]} & 0 \\
1 & \frac{\alpha^{2} \alpha_{2}}{\left[\alpha_{2}\right]} & \frac{\alpha^{3} \alpha_{2}^{2} \alpha_{3}}{\left[\alpha_{2}\right]^{3 / 2}\left[\alpha_{2} \alpha_{3}\right]} \\
\frac{\alpha \beta^{2} \beta_{2}^{2} \beta_{3}}{\left[\beta_{2}\right]^{3 / 2}\left[\beta_{2} \beta_{3}\right]} & 1 & \frac{\alpha}{\left[\alpha_{2}\right]} \\
0 \quad \frac{\alpha \beta^{2} \beta_{2}^{2} \beta_{3}}{\left[\beta_{2}\right]^{3 / 2}\left[\beta_{2} \beta_{3}\right]} & 1 & \alpha
\end{array}\right|= \\
= \\
+2 \frac{a_{-1}^{4}\left(\alpha^{4}-3 \frac{\alpha^{4} \alpha_{2}}{\left[\alpha_{2}\right]}+\frac{\alpha^{4} \alpha_{2}^{2}}{\left[\alpha_{2}\right]^{2}}+\frac{\alpha^{8} \beta^{4} \alpha_{2}^{8} \alpha_{3}^{2} \beta_{3}^{2}}{\left[\alpha_{2}\right]^{6}\left[\alpha_{2} \alpha_{3}\right]^{2}\left[\beta_{2} \beta_{3}\right]^{2}}+\right.}{\left[\alpha_{2}\right]^{\frac{7}{2}}\left[\beta_{2} \beta_{3}\right]}+2 \frac{\alpha^{4} \alpha_{2}^{2} \alpha_{3}}{\left[\alpha_{2}\right]^{\frac{3}{2}}\left[\alpha_{2} \alpha_{3}\right]}-2 \frac{\alpha^{6} \beta^{2} \alpha_{2}^{4} \beta_{3} \alpha_{3}}{\left[\alpha_{2}\right]^{3}\left[\alpha_{2} \alpha_{3}\right]\left[\beta_{2} \beta_{3}\right]}- \\
\left.-2 \frac{\alpha^{6} \beta^{2} \alpha_{2}^{5} \alpha_{3} \beta_{3}}{\left[\alpha_{2}\right]^{4}\left[\alpha_{2} \alpha_{3}\right]\left[\beta_{2} \beta_{3}\right]}\right)=: a_{-1}^{4} \alpha^{4} J\left(\alpha_{3}, \beta_{3}\right),
\end{gathered}
$$

the inequality $J\left(\alpha_{3}, \beta_{3}\right)<0$ yields $f(z) \notin P F_{4}$.

Substituting $\beta=\left(1+\alpha_{2}\right) /\left(\alpha_{2} \alpha\right)$ into $J\left(\alpha_{3}, \beta_{3}\right)$, we have

$$
\begin{gathered}
J\left(\alpha_{3}, \beta_{3}\right)=1-3 \frac{\alpha_{2}}{[\alpha]}+\frac{\alpha_{2}^{2}}{[\alpha]^{2}}+\frac{\alpha_{2}^{4} \alpha_{3}^{2} \beta_{3}^{2}}{\left[\alpha_{2}\right]^{2}\left[\alpha_{2} \alpha_{3}\right]^{2}\left[\beta_{2} \beta_{3}\right]^{2}}+ \\
+2 \frac{\alpha_{2}^{2} \beta_{3}}{\left[\alpha_{2}\right]^{\frac{3}{2}}\left[\beta_{2} \beta_{3}\right]}+2 \frac{\alpha_{2}^{2} \alpha_{3}}{\left[\alpha_{2}\right]^{\frac{3}{2}}\left[\alpha_{2} \alpha_{3}\right]}-2 \frac{\alpha_{2}^{2} \beta_{3} \alpha_{3}}{\left[\alpha_{2}\right]\left[\alpha_{2} \alpha_{3}\right]\left[\beta_{2} \beta_{3}\right]}- \\
-2 \frac{\alpha_{2}^{3} \alpha_{3} \beta_{3}}{\left[\alpha_{2}\right]^{2}\left[\alpha_{2} \alpha_{3}\right]\left[\beta_{2} \beta_{3}\right]}=: J(0,0)+\alpha_{3} J_{1}+\beta_{3} J_{2} .
\end{gathered}
$$

In all fractions entering in the last equations the numerators do not exceed 1 , while the denominators are greater than 1 . Therefore, $\left|J_{1}\right|+\left|J_{2}\right| \leq 9$. The quantity $\alpha_{3} J_{1}+\beta_{3} J_{2}$ may be made arbitrarily small by choosing sufficiently 
small $\alpha_{3}>0$ and $\beta_{3}>0$. Therefore, the signs of $J\left(\alpha_{3}, \beta_{3}\right)$ and $J(0,0)$ coincide for all sufficiently small $\alpha_{3}>0$ and $\beta_{3}>0$. Note that

$$
J(0,0)=1-3 \frac{\alpha_{2}}{\left[\alpha_{2}\right]}+\frac{\alpha_{2}^{2}}{\left[\alpha_{2}\right]^{2}}=-\frac{\alpha_{2}^{2}+\alpha_{2}-1}{\left[\alpha_{2}\right]^{2}}<0, \quad \text { since } \quad \alpha_{2}>\frac{-1+\sqrt{5}}{2} .
$$

Acknowledgment. The author would like to thank Professors I. V. Ostrovskii and C. Y. Yıldırım for helpful suggestions and comments.

\title{
References
}

[1] A. EDREI, On the generating function of a doubly infinite totally positive sequence, Trans. Amer. Math. Soc., 74(1953), 367-383.

[2] S. Karlin, Total positivity, Stanford University Press (Stanford, 1968).

[3] I. V. Ostrovski and N. A. Zheltukhina, On power series having sections with multiply positive coefficients and a theorem of Pólya, J. London Math. Soc., 58(1998), 97-110.

[4] I. V. Ostrovskin and N. A. Zheltukhina, Parametric representation of a class of multiply positive sequences, Complex Variables, 37(1998), 457-469.

[5] I. J. Schoenberg, Some analytical aspects of the problem of smoothing, Courant Anniversary Volume, (New York, 1948), 351-370.

[6] I. J. SchoenberG, On the zeros of generating functions of multiply positive sequences and functions, Ann. Math., 62(1955), 447-471.

\section{О рядах Лорана с многократно положительными коэффициентами}

\author{
Н. А. ЖЕЛТУКИНА
}

Рассмотрен класс последовательностей $\left\{a_{k}\right\}_{k=-\infty}^{\infty}$, отрезки которых $\left\{a_{k}\right\}_{k=-n}^{n}$ являются 3 -кратно положительными в смысле Полиа-Фекете при всех $n=1,2, \ldots$, и $a_{0} \neq 0$. Получено описание этого класса в терминах независимых параметров. Найдены оценки роста соответствующих рядов Лорана $\sum_{k=-\infty}^{\infty} a_{k} z^{k}$.

DEPARTMENT OF MATHEMATICS

BILKENT UNIVERSITY

06800 ANKARA

TURKEY

e-mail: natalya@fen.bilkent.edu.tr 